\title{
Effect of Gamma Irradiation on Photoelectric Parameters of Double- Barrier Structure Based on Silicon
}

\section{Abasov FP*}

Institute of Radiation Problems of Azerbaijan National Academy of Sciences, Russia

\begin{abstract}
Developed a silicon-based photodetector with high sensitivity integrated in the short range. The influence of gamma radiation on the mechanism of current flow in the structure type Schottky barrier, and the p-n junctions. It is shown that the double-barrier structure can improve the photoelectric parameters of conventional detectors.
\end{abstract}

Keywords: Silicon photo-detectors; Photo-converters; Doublebarriers structure; Ionizing radiation

\section{Introduction}

Silicon photodetectors, still the most common type of photoconverters. One of the main ways of increasing the speed and increase the spectral sensitivity of modern detectors with one transition is the creation of multi-barrier structures. Reliability of the resulting structures at elevated conditions of radiation, as detectors of ionizing radiation is an important task is the subject of our research [1,2].

Recently, to extend the spectral sensitivity range commonly used methods [3,4] leads to an increase of the photocurrent in the wavelength region of the spectrum. Examples are Graded band gap semiconductors structure; dragging fields, etc., based on the reduction of the surface recombination velocity. In our case, such a possibility, but you can create a planar version of the field due to the $p-n$ junction is included in the opposite direction (Figure 1).

\section{Experimental Results and Discussion}

First obtained and investigated characteristics of double-barrier structures created on the same plane have shown advantages over conventional structures. With the introduction of the second barrier it increases the integral sensitivity in photodiode $(\beta+1)$ times speed and 300 times in the structure. Non-ideality coefficient $\beta=1.35$ is small, due to the presence of an electrical field that is due to the drift mechanism in contact with the environment. UV radiation, for example, the case is being investigated jointly with the celebration structure. Quanta gamma irradiation of UV radiation in Figure 2 and then irradiated samples photo response annealing limit and display the results. As can be seen, the sensitivity of the barrier structure in Figure 3 .

The optimal radiation dose exposure increases the tension in the region near UF reduced. Annealing small and medium-sized doses of radiation defects are observed. Annealing high doses of radioactivity to the defects observed defects in the radiation resistant to the establishment of the conductivity probe is decreasing. In this study, two types of planar Schottky barrier fenced structure of $p-n$ transitions and are being studied and a sample of gamma radiation used to record the conditions of radiation shown UF [5].

To create photo detectors planar design with internal amplification created Schottky barrier Au-Si. The starting material used in the structure of $\mathrm{p}$-n-type on the silicon substrate. To create photodetectors planar design with internal amplification created Schottky barrier Au$\mathrm{Si}$. The starting material used in the structure of $\mathrm{p}$-n-type on the silicon substrate. The control of current conducted by the light selection voltage $\mathrm{K}-\mathrm{E}$ so that the collector junction is closed and the open-emitter, with the free base. Under the influence of light therein, electrons and holes are generated. At the collector transition yes there is a division of electrondy-term couples reached after consequence diffusion boundary return. Holes are moved to the collector field transition, increased own current and the electrons remain in the database, reducing its potential. When
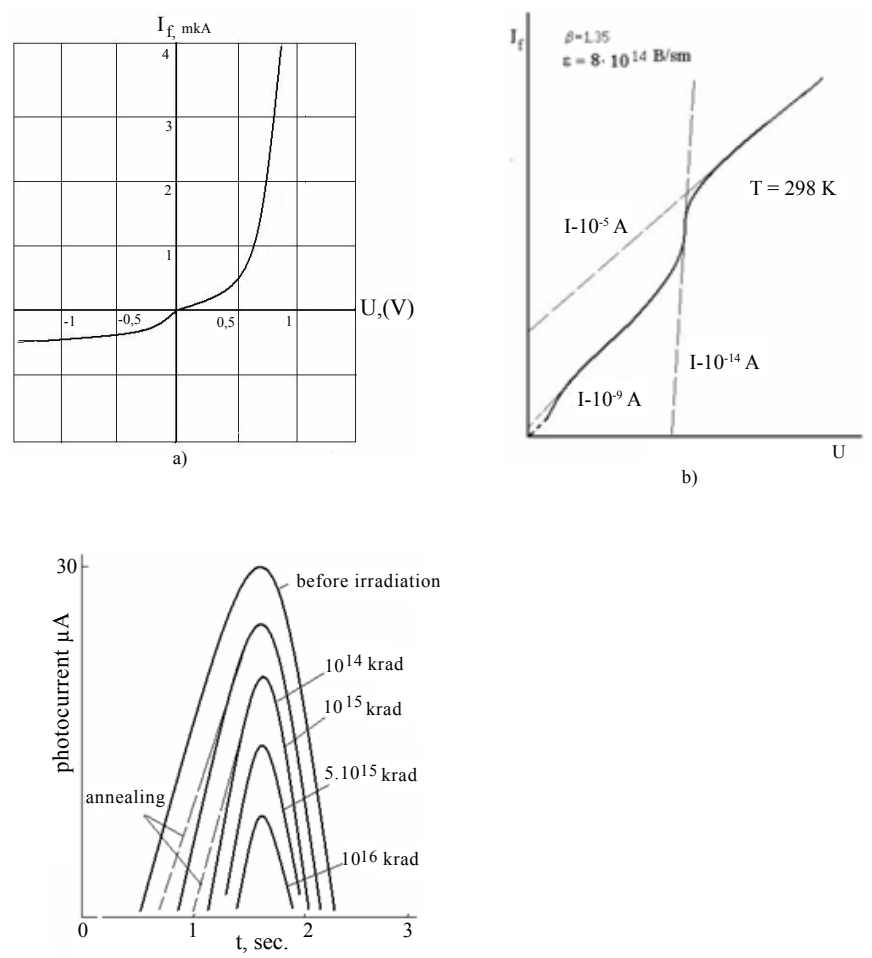

c)

Figure 1: a) and b) Volt-Ampere characteristics, c) photo response.

*Corresponding author: Abasov FP, Institute of Radiation Problems of Azerbaijan National Academy of Sciences, AZ 1143, Baku, str. B.Vaqabzade 9, Russia, Tel: 994125393391; E-mail: fpabasov@mail.ru

Received June 09, 2016; Accepted July 13, 2016; Published July 23, 2016

Citation: Abasov FP (2016) Effect of Gamma Irradiation on Photoelectric Parameters of Double-Barrier Structure Based on Silicon. J Material Sci Eng 5: 269. doi:10.4172/2169-0022.1000269

Copyright: (c) 2016 Abasov FP. This is an open-access article distributed under the terms of the Creative Commons Attribution License, which permits unrestricted use, distribution, and reproduction in any medium, provided the original author and source are credited. 


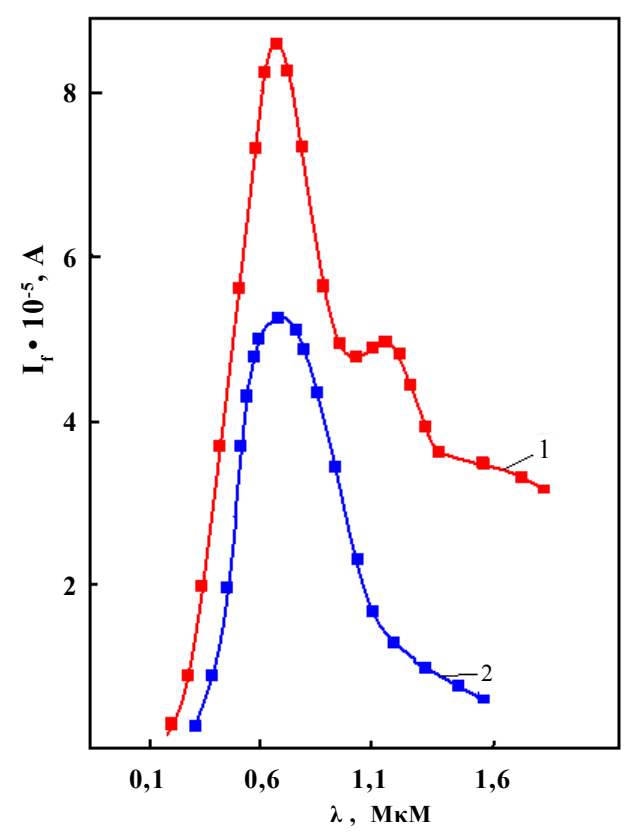

Figure 2: Spectral characteristic of the double-barrier structures a) to radiation $1 .-\mathrm{U}_{\text {rev. }}=0 \mathrm{~V} ; 2 .-\mathrm{U}_{\text {rev. }}=0.5 \mathrm{~V} ; \mathrm{T}=300 \mathrm{~K}$.

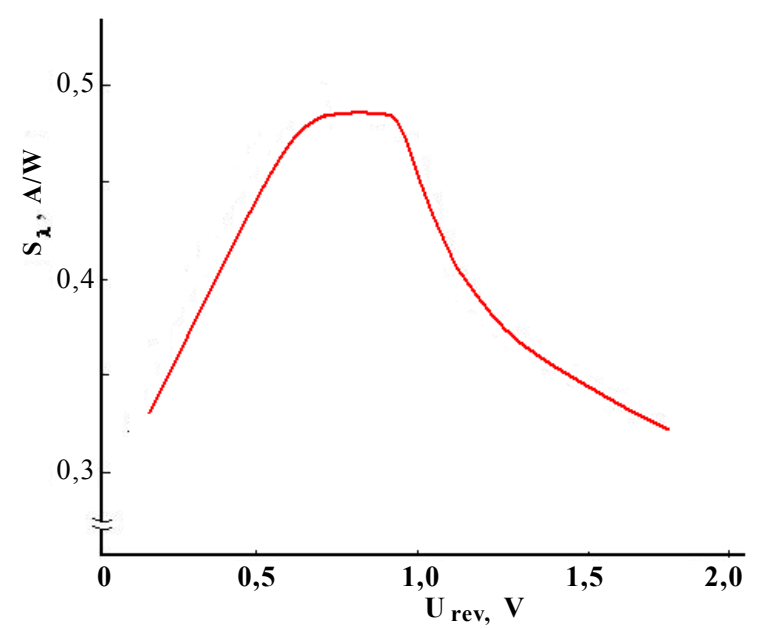

Figure 3: Dependence of the spectral sensitivity of the structure the applied reverse bias the $p-n$ junction.

this transition occurs at the emitter additional direct voltage, which increases the injection of holes from the emitter into the base. Injected hole, reaching the collector junction causes an additional increase in the collector current. Since the total collector current is proportional to the ratio of internal amplification, we have an increase of spectral sensitivity, reaching $0.65 \mathrm{~A} /$ watt [6].

With further increase $U_{\text {con }}$. spektral sensitivity decreases. This behavior $S_{\lambda}$ due to increased space charge region and improving the collection coefficient photocarriers. With further growth $\mathrm{U}_{\text {con }}$, because of the overlapping zones, blocked photoinjection BSh structure and operates on a single photodiode (Figure 3 ). The structure was irradiated at $\mathrm{T}=300 \mathrm{~K}{ }^{60} \mathrm{Co}$ gamma rays. Isochronous $(30 \mathrm{~min})$ annealing of radiation defects was conducted in the temperature range $\mathrm{Ta}=200$ $450 \mathrm{~K}$.
Method photovoltage $\mathrm{Voc}=\ln +\ln =\mathrm{Vj}+\mathrm{VB}$ showed that the primary radiation defects $(\mathrm{RD})$ in the p-Si crystals at $300 \mathrm{~K}$ are positively charged. CVC analysis (Figure 4) and spectral characteristics showed that recombination currents increase with increasing irradiation dose. Annealing diodes reduces recombination currents.

At a temperature $\mathrm{Ta} \approx 300^{\circ} \mathrm{C}$ annealing and rearrangement occurs to form complexes divacancies $\mathrm{V} 2+\mathrm{O}$, and at $\mathrm{Ta}=350^{\circ} \mathrm{C}$ annealed active A-centers $(\mathrm{V}+\mathrm{O})$ and complexes $\left(\mathrm{V}_{2}+\mathrm{O}\right)$. Analysis of changes in current flow through the BS and the pn junction showed the difference effect of annealing near the surface and inside the crystal. This can be explained by the increase of the photo response BS associated with charge accumulation and improving the collection coefficient. Figure 5 shows the spectral dependence of photocurrent curves before and after gamma irradiation at different doses and after annealing at $\mathrm{T}$ $=400^{\circ} \mathrm{C}$ for $30 \mathrm{~min}$. Annealing affects the spectral characteristics

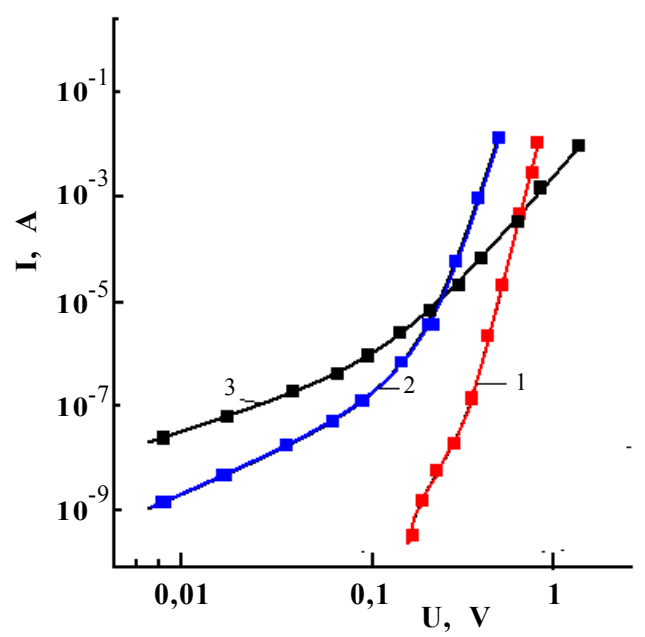

Figure 4: Volt-ampere characteristics of p-n-junction 1.original. 2. Dy $=100$ krad. 3. Dy $=200 \mathrm{krad}$. Annealing results are insignificant.

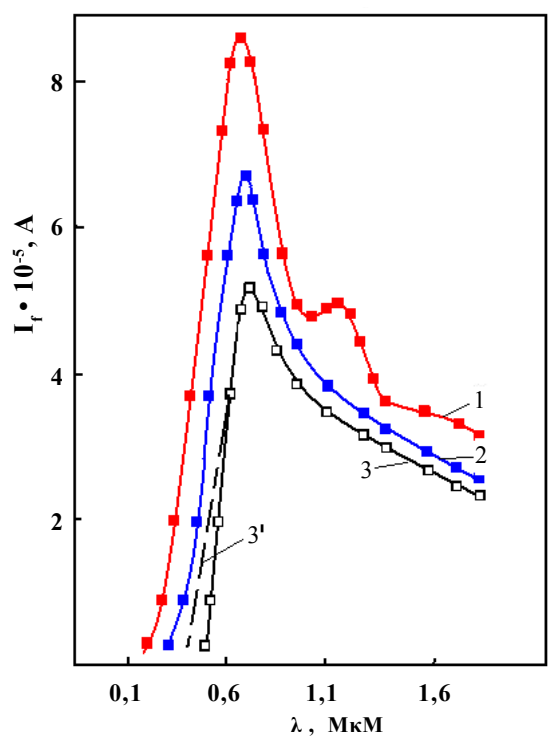

Figure 5: Spectral characteristic double-barrier structure after irradiation with gamma rays: 1-up irradiation, 2-dose 150 krad., 3) dose of 200 krad., 3 ') annealed at $\mathrm{T}=400^{\circ} \mathrm{C}$ for $30 \mathrm{~min}$ 
Citation: Abasov FP (2016) Effect of Gamma Irradiation on Photoelectric Parameters of Double-Barrier Structure Based on Silicon. J Material Sci Eng 5: 269. doi:10.4172/2169-0022.1000269

Page 3 of 3

significantly. With increasing doses of gamma irradiation growth of the photocurrent decreases.

\section{Conclusions}

Thus, it can be argued that the main role in the electrical losses studied silicon structures play oxygen centers $\left(\mathrm{V}_{2}+\mathrm{O}\right.$ and $\left.\mathrm{V}+\mathrm{O}\right)$. With increasing irradiation dose, and the annealing temperature increases, especially $\mathrm{CVC}$ and due to the change of spectral characteristics resistance $\mathrm{n}-\mathrm{Si}$ (the base region of the structure) caused by the accumulation (increasing dose) and the disappearance or rearrangement (for annealing), radiation-induced defects. Known that the defect capture rate electrons and (or) the hole in the first place depends on the capture cross section and the position of the energy level in the forbidden band. These parameters are essentially the "individual" characteristic defect [7-9]. Upon annealing, the structure is changing the point of radiation defects and their disappearance. In this case mainly the accumulation of similar defects. Comparison with literature data shows that the main role in the photovoltaic losses of these structures play an oxygen centers $\left(\mathrm{V}_{2}+\mathrm{O}\right.$ and $\left.\mathrm{V}+\mathrm{O}\right)$. With further increase of radiation dose an irreversible reduction of photosensitivity due to a significant increase in the resistance base.

\section{References}

1. Sze SM, Ng KK (2006) Physics of Semiconductor Devices. New York, Wiley.

2. Bakaleynikov LA, Flegontova EJ, Pogrebitsky K, Eremin IV (2004) Theoretical concepts of operation of a semiconductor detector based on a $\mathrm{p}$-n junction. Technical Physics 49: 1181-1190.

3. Savchenko IV (1985) Theoretical Foundations of dosimetry.

4. Kosyachenko LA, Maslyanchuk EL, Rarenko IM, Sklyarchuk VM (2004) Issledoanie carrier collection CdZnTe-ray detectors and - radiation photoelectrically. Semiconductors 388: 1018.

5. Madatov RS, Mehrabova MA, Abasov FP (2006) Fast acting detectors for $\mathrm{Y}$ quantums on the Au-Si. The IV euroasian conference on nuclear science and its application. Baku, Azerbaijan.

6. Bustos E, Godínez LA (2011) Modified surfaces with nano-structured composites of prussian blue and dendrimers. New materials for advanced electrochemical applications. Int J Electrochem Sci 6: 1-36.

7. Abasov FP (2014) Preparation of thin films to create solar cells. Photonics 2 : 72-90.

8. Abasov FP (2014) Influence of gamma radiation on electrik propertien of silicon solar cells. International Journal of Pure and Applied Science and Technology 21: 12-16.

9. Abasov FP, Mustafayev YM (2014) Effect of gamma irradiation on electrophysical and photoelectric parameters of double-barrier structure based on silicon. IAEA, INIS. 